\title{
Perfil clínico-epidemiológico de las defunciones por influenza con antecedente de vacunación oportuna, México 2010-2018
}

\author{
Pablo Antonio Kuri-Morales, ${ }^{1}$ Guadalupe Díaz del Castillo-Flores, ${ }^{2 *}$ Andrés Castañeda-Prado ${ }^{1}$ y \\ Salma Rosario Pacheco-Montes ${ }^{3}$ \\ ${ }^{1}$ Universidad Nacional Autónoma de México, Facultad de Medicina, Ciudad de México; ${ }^{2}$ Servicios de Salud de Veracruz, Subdirección de Prevención \\ y Control de Enfermedades, Veracruz; ${ }^{3}$ Secretaría de Salud, Dirección General de Epidemiología, Ciudad de México. México
}

\section{Resumen}

Introducción: Las epidemias de influenza son de mayor riesgo en los extremos de la vida y en personas con comorbilidades. La vacunación efectiva previene la aparición de casos graves y disminuye la mortalidad. Objetivo: Describir las defunciones por influenza en México con antecedente de vacunación oportuna, de 2010 a 2018. Método: Estudio transversal descriptivo en el que se utilizó la base de datos del Sistema de Vigilancia Epidemiológica de Influenza. Resultados: De 2010 a 2018 fallecieron por influenza 65 personas con vacunación, $55 \%(n=36)$ de las cuales por tipo A (H1N1), $51 \%$ ( $n=33)$ del sexo femenino, la mediana de edad fue de 57 años, $21 \%(n=14)$ no cumplía la definición operacional de enfermedad tipo influenza o infección respiratoria aguda grave, $83 \%(n=54)$ tenía al menos una comorbilidad; las comorbilidades más frecuentes fueron diabetes mellitus e hipertensión arterial (32\% cada una); $55 \%(n=36)$ recibió tratamiento antiviral y solo $8 \%$ ( $n=5$ ) no presentaba comorbilidades y tenía tratamiento con oseltamivir. Conclusiones: Las defunciones por influenza con vacunación oportuna representan un porcentaje muy bajo del total. La vacunación contra influenza ha sido una estrategia de prevención específica que disminuye la carga de la enfermedad.

PALABRAS CLAVE: Influenza humana. Causa de defunción. Vacunación.

\begin{abstract}
Introduction: Influenza epidemics are of higher risk at the extremes of life and in people with comorbidities. Effective vaccination prevents the occurrence of serious cases and decreases mortality. Objective: To describe deaths from influenza with a history of timely vaccination, from the 2010 to the 2018 season in Mexico. Method: Cross-sectional, descriptive study where the Influenza Epidemiological Surveillance System database was used. Results: From 2010 to 2018, 65 vaccinated individuals died from influenza, from which $55 \%$ of cases $(n=36)$ were due to type $A(H 1 N 1), 51 \%(n=33)$ were females, median age was 57 years, $21 \%(n=14)$ did not meet the operational definition of influenza-like illness or severe acute respiratory infection, $83 \%(n=54)$ had at least one comorbidity, with the most common being diabetes mellitus and hypertension (32\% each); $55 \%(n=36)$ of deaths received antiviral treatment and only $8 \%(n=5)$ had no comorbidities and received treatment with oseltamivir. Conclusions: Deaths from influenza with timely vaccination represent a very low percentage of the totality. Vaccination against influenza has been a specific prevention strategy that decreases disease burden.
\end{abstract}

KEY WORDS: Human influenza. Cause of death. Vaccination.

Correspondencia:

*Guadalupe Díaz del Castillo-Flores

E-mail: guadelupediazdelcastilloflores@gmail.com
Fecha de recepción: 26-09-2018

Fecha de aceptación: 21-02-2019

DOI: 10.24875/GMM.19004708
Gac Med Mex. 2019;155:457-463

Disponible en PubMed www.gacetamedicademexico.com 


\section{Introducción}

La influenza, estacional o pandémica, es una enfermedad causada por un virus ARN perteneciente a la familia Orthomyxoviridae. Existen tres tipos de virus de influenza: A, B y C, que a su vez incluyen diferentes subtipos. La transmisión del virus es por vía aérea o mediante fómites y tiene alto potencial pandémico., ${ }^{1,2}$

Los virus de la influenza $A$ están divididos en subtipos según la caracterización de dos antígenos de superficie: hemaglutinina y neuroaminidasa. Los virus de la influenza A (H1N1 y H3N2) y B circulan simultáneamente en el mundo. Las nuevas variantes del virus de la influenza aparecen como resultado de mutaciones puntuales y eventos de recombinación que ocurren durante la replicación viral, generando variaciones antigénicas frecuentes. ${ }^{2}$

Los subtipos nuevos o muy diferentes del virus de la influenza $A$ resultantes de las variaciones antigénicas tienen el potencial de producir una pandemia, desde el momento en que son capaces de causar enfermedad en humanos, mantener una transmisión sostenida efectiva y al existir poca o ninguna inmunidad previa en la población. ${ }^{2}$

Las epidemias de influenza pueden afectar seriamente a la población y son de mayor riesgo en los extremos de la vida y en personas con comorbilidades. A lo largo de la historia, la influenza ha ocasionado grandes epidemias que se han traducido en altas tasas de mortalidad. La vigilancia epidemiológica de la influenza y la vacunación son indispensables para la prevención de brotes y epidemias que pueden poner en riesgo la vida de la población. La vacunación efectiva previene la aparición de casos graves y disminuye la mortalidad. ${ }^{1,3}$

La eficacia y efectividad de la vacuna contra la influenza dependen de numerosos factores como edad, inmunocompetencia, grado de similitud entre los virus contenidos en la vacuna y virus en circulación, entre otros (Tabla 1). ${ }^{4}$

Tras la vacunación, los niveles de las proteínas virales hemaglutinina y neuroaminidasa disminuyen lentamente; la reducción podría ser superior a $50 \%$ a los 600 días. ${ }^{3,5}$ En adultos mayores de 60 años se ha reportado la seroprotección tras la vacunación contra influenza tipo A; en los componentes H3N2 y H1N1 ha sido mayor a cuatro meses. ${ }^{6}$ Un estudio en España concluyó que las personas con antecedente de vacunación mayor a los 200 días tienen mayor riesgo de contraer la enfermedad que quienes lo

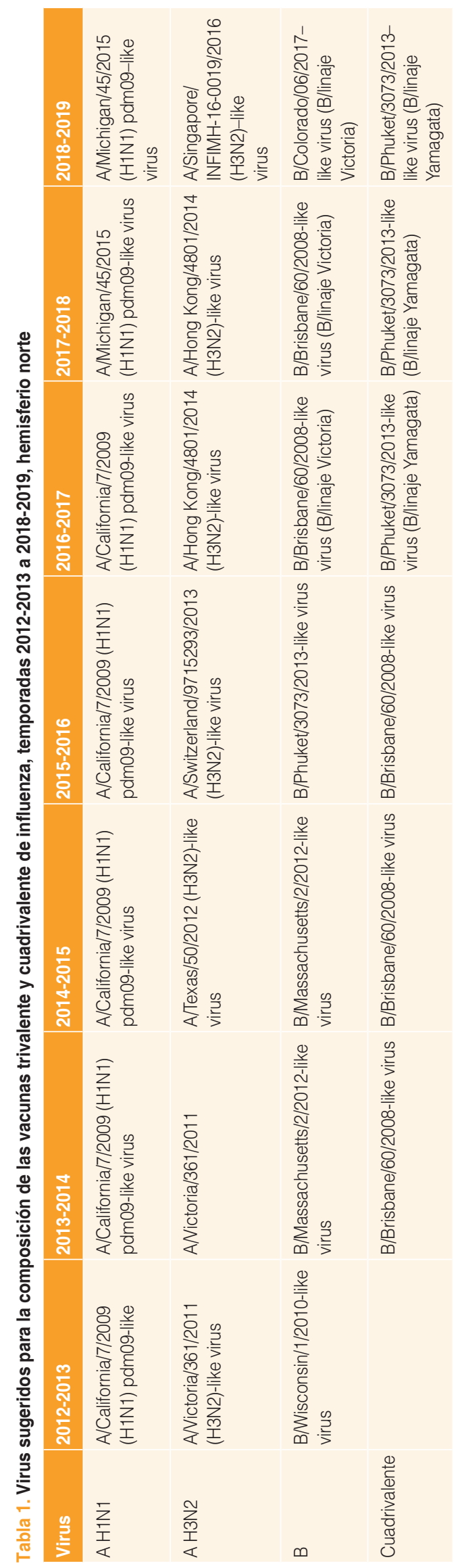


hicieron en los últimos días. ${ }^{7}$ En Reino Unido, el rango de efectividad en la temporada 2011-2012 para influenza A (H3N2) se calculó en $53 \%$ en las personas vacunadas menos de tres meses antes y en $12 \%$ en las personas vacunadas tres o más meses antes. ${ }^{8}$

El tratamiento para influenza consiste en inhibidores de la neuraminidasa: oseltamivir y zanamivir. Los ensayos clínicos y los datos observacionales muestran que el tratamiento antiviral temprano puede acortar la duración de los síntomas de fiebre y enfermedad y reducir el riesgo de complicaciones. ${ }^{9}$

En México, la vigilancia epidemiológica de influenza es convencional y centinela, de acuerdo con los estándares recomendado por la Organización Mundial de la Salud. El sistema de vigilancia especial se denomina SISVEFLU (Sistema de Vigilancia Epidemiológica de Influenza) y forma parte del Sistema Nacional de Vigilancia Epidemiológica. El SISVEFLU cuenta con 559 unidades de salud monitoras de influenza (USMI) de primer, segundo y tercer nivel de atención del Sistema Nacional de Salud en México, distribuidas en las 32 entidades federativas del país. El objetivo principal de SISVEFLU es vigilar el tipo de agente etiológico que circula y produce infección respiratoria aguda grave (IRAG) en México e identificar casos nuevos de enfermedad respiratoria asociada con la presencia de nuevos agentes o el incremento de influenza estacional. ${ }^{10}$

La vigilancia epidemiológica de influenza incluye las siguientes definiciones:

- Enfermedad tipo influenza (ETI): persona de cualquier edad que presente o refiera haber tenido fiebre mayor o igual a $38{ }^{\circ} \mathrm{C}$, tos y cefalea acompañadas de uno o más de los siguientes signos o síntomas: rinorrea, coriza, artralgias, mialgias, postración, odinofagia, dolor torácico, dolor abdominal, congestión nasal o diarrea.

- IRAG: persona de cualquier edad que presente dificultad al respirar, con antecedente de fiebre mayor o igual a $38^{\circ} \mathrm{C}$ y tos, o uno o más de los siguientes síntomas: ataque al estado general, dolor torácico o polipnea.

- Defunción por influenza: paciente fallecido que haya cumplido con la definición operacional de ETI/IRAG y que cuente con resultado positivo a influenza emitido por uno de los laboratorios avalados por la Red Nacional de Laboratorios de Salud Pública, y que en su certificado de defunción contenga como causa básica el diagnóstico de influenza o neumonía. Una defunción por influenza está sujeta a vigilancia epidemiológica, por lo que necesariamente debe ser ratificada o rectificada por la metodología del Sistema Epidemiológico y Estadístico de las Defunciones. ${ }^{11}$

El objetivo de este estudio es caracterizar las defunciones por influenza con antecedente de vacunación ratificadas por el Sistema Epidemiológico y Estadístico de las Defunciones de las temporadas 2010-2011 a 2017-2018 en México.

\section{Método}

Estudio transversal descriptivo en el que se utilizó la base de datos del SISVEFLU de los años 20102018. Las variables incluidas forman parte del estudio de caso de influenza: edad, sexo, embarazo, entidad de residencia, ocupación, fecha de ingreso a la unidad de atención, fecha de inicio de síntomas, comobilidad, diagnóstico probable, tratamiento con antivirales, tipo de antiviral, antecedente de vacuna contra influenza, fecha de vacunación contra influenza, si se desconoce antecedente de vacunación, toma de muestra, resultado de la muestra y fecha de defunción.

Inicialmente se incluyeron todas las defunciones por influenza de 2010 a 2018; posteriormente se excluyeron aquellas sin antecedente de vacunación, con antecedente desconocido o sin registro de la fecha de vacunación contra influenza. Adicionalmente se excluyeron los registros con fecha de vacunación menor 0 igual a un día (ningún enfermo grave fue vacunado durante su atención) o mayor de 600 (después de este tiempo los anticuerpos se reducen por debajo de $50 \%$ ).

Se seleccionaron las defunciones con antecedente de vacunación correspondiente a la temporada de cada uno de los años analizados con al menos 21 días (tiempo promedio de respuesta inumne inducida por la vacunación) y se excluyeron aquellas cuya fecha de vacunación correspondía a otra temporada (Figura 1).

\section{Resultados}

De la semana epidemiológica 40 de 2010 hasta la 20 de 2018 ocurrieron 3089 defunciones por influenza, solo 65 defunciones ( $2.1 \%$ del total) fueron reportadas con antecedente de vacunación con la vacuna de la temporada y con al menos 21 días desde la vacunación hasta el inicio de síntomas de influenza (Figura 1). En enero de 2014 se registró el mayor número de defunciones, $17 \%(n=11)$ (Figura 2). El promedio de días desde la vacunación hasta el inicio de síntomas fue de $78 ; 88 \%(n=57)$ de las defunciones contaba con registro de fecha de defunción. El promedio de días desde el inicio de los síntomas 


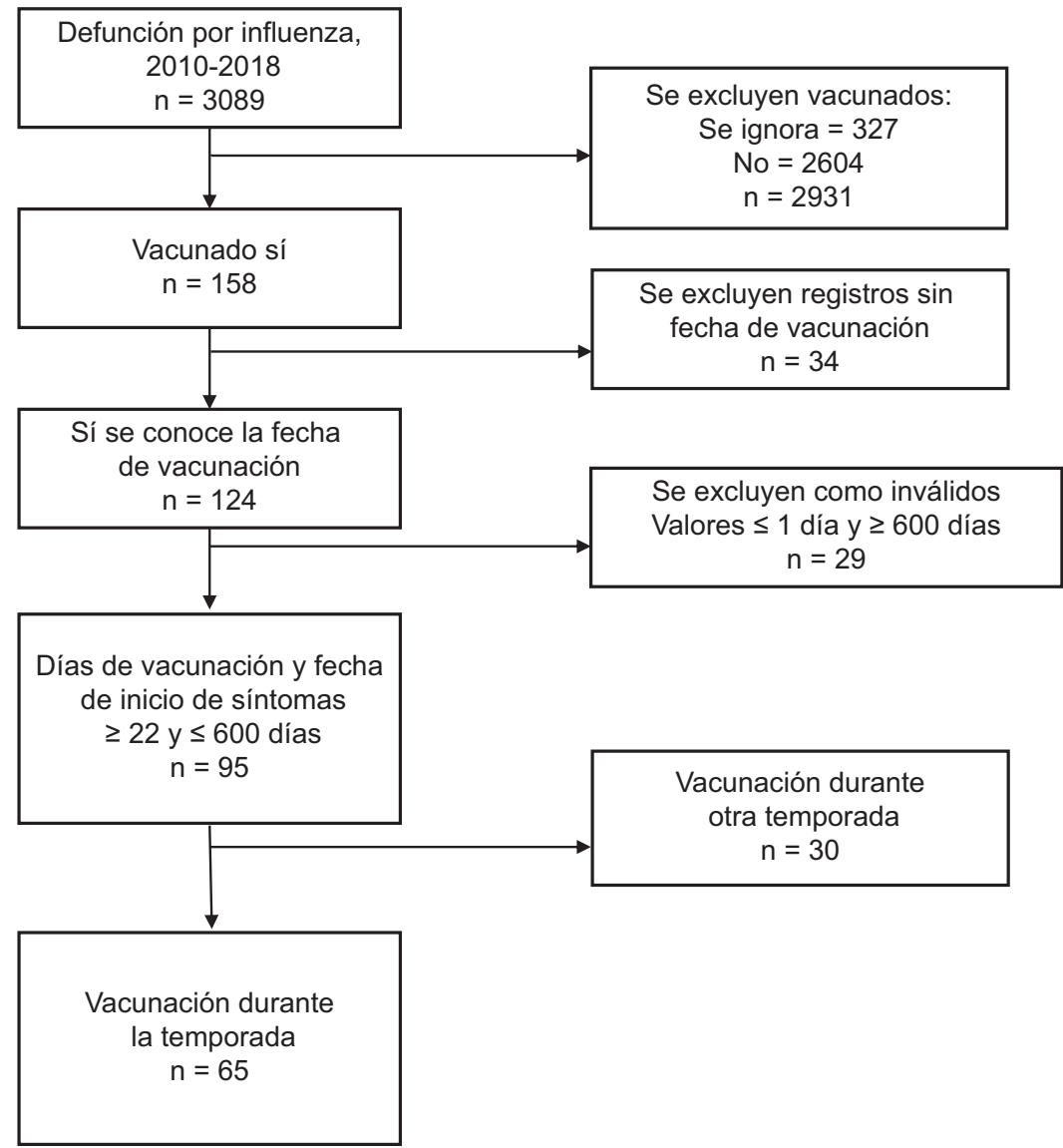

Figura 1. Diagrama de flujo del proceso de selección de las defunciones con antecedente de vacunación oportuna contra influenza.

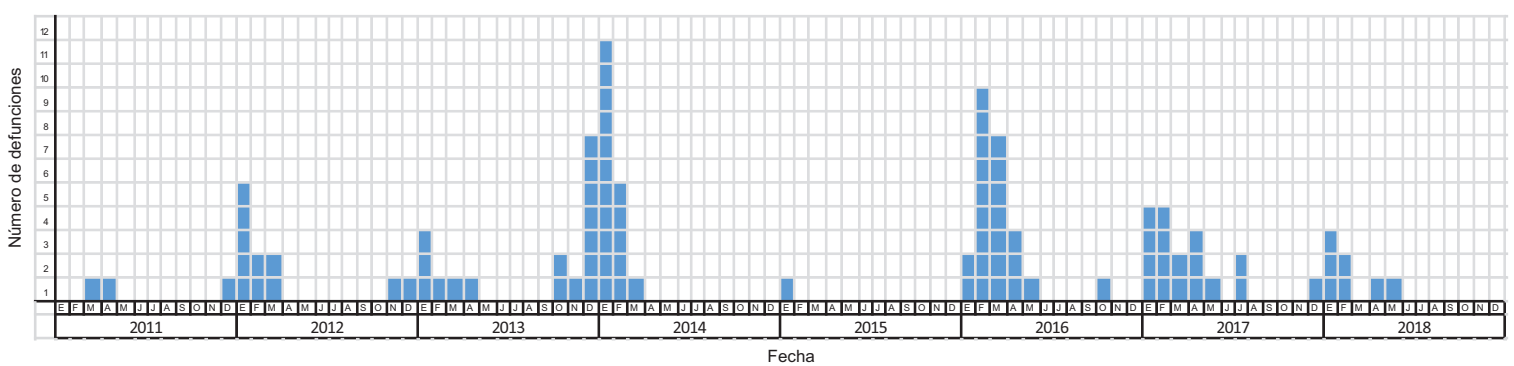

Figura 2. Temporalidad de las defunciones por influenza con antecedente de vacunación, México 2010-2018 ( $n=65)$.

hasta la fecha de defunción fue de 10.3 (mediana de 9 y rango de 0-37).

La mayoría de las defunciones $(n=36)$ fue por el subtipo $A(H 1 N 1) ; 15$ por $A(H 3)$, ocho por influenza $B$ $y$ dos no estaban subtipificadas (Figura 3 ).

\section{Caracterización sociodemográfica}

La totalidad de las defunciones $(n=65)$ fueron notificadas por las unidades de salud monitoras de influenza. De acuerdo con el sitio de ocurrencia, $17 \%$ $(n=11)$ de las defunciones correspondió a la Ciudad de México, $12 \%(\mathrm{n}=8)$ al Estado de México, $12 \%$ $(n=8)$ a Jalisco, $9 \%(n=6)$ a Michoacán y $6 \%(n=4)$ a Veracruz. La distribución por sexo fue muy similar: $51 \%(n=33)$ fue del sexo femenino. La mediana de edad fue de 57 años con rango de 1 a 94. La ocupación más frecuente fue el hogar, $32 \%(n=21)$.

\section{Definición operacional: ETI/IRAG}

El $21 \%(n=14)$ de las 65 defunciones no cumplieron con la definición operacional de ETI o IRAG a pesar de contar con resultado final positivo para 


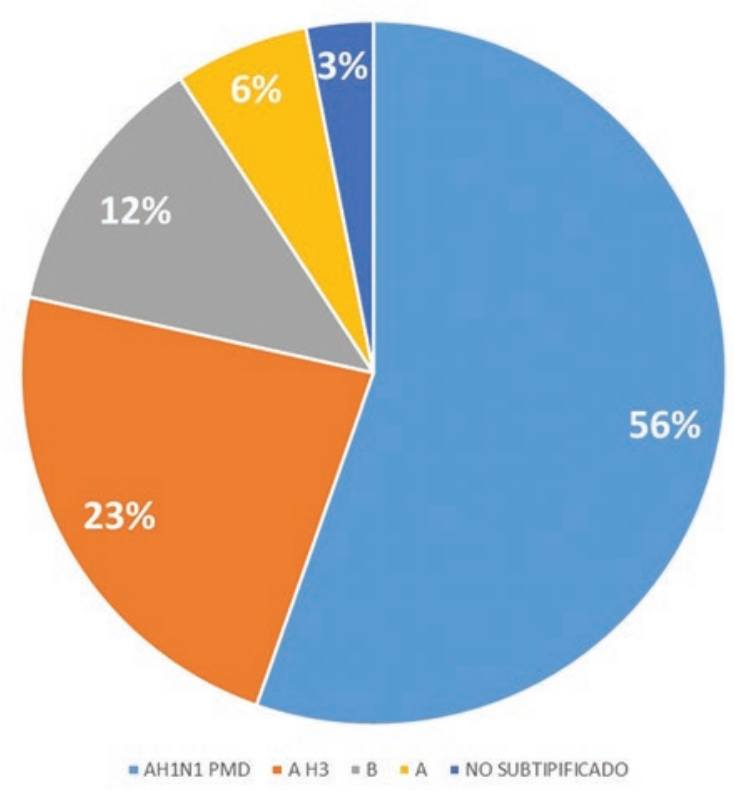

Figura 3. Distribución de las defunciones por influenza con antecedente de vacunación por subtipo, México 2010-2018 ( $n=65)$.

influenza. Las cinco defunciones en menores de cinco años cumplieron $100 \%$ la definición operacional. De las 32 defunciones en el grupo de seis a 64 años, siete $(22 \%)$ no cumplieron con la definición operacional de ETI o IRAG y de las 28 defunciones mayores de 65 años, siete (25\%) tampoco. Consideramos que condiciones inherentes a los enfermos determinaron que 14 defunciones no cumplieran estrictamente con la definición operacional, sin embargo, ante la sospecha del clínico se tomaron muestras, las cuales resultaron positivas.

\section{Comorbilidades}

En $83 \%(n=54)$ de las defunciones existía al menos una comorbilidad, en $29 \%(n=19)$ una, en $26 \%$ $(n=17)$ dos, en $18 \%(n=12)$ tres, en $8 \%(n=5)$ cuatro y en $1.5 \%(n=1)$ cinco. De los menores de cinco años, solo uno presentaba una comorbilidad, registrada como "otra" (Tabla 2).

\section{Tratamiento antiviral}

Solo $55 \%$ de las defunciones $(n=36)$ recibió tratamiento antiviral, 35 oseltamivir y uno zanamivir. De estas, únicamente cinco tenían el registro de fecha de inicio de tratamiento; $6 \%$ de las defunciones $(n=4)$ tenía registrado haber iniciado el tratamiento en las primeras 72 horas después del inicio de los síntomas; en un caso se registró inicio antiviral en el
Tabla 2. Frecuencia de comorbilidades en las defunciones por influenza con antecedente de vacunación, México 2010-2018 $(n=54)$

\begin{tabular}{|l|l|l|}
\hline Comorbilidad & n & $\%$ \\
\hline Diabetes mellitus & 21 & 32 \\
\hline Hipertensión arterial & 21 & 32 \\
\hline Obesidad mórbida & 15 & 23 \\
\hline Insuficiencia renal crónica & 12 & 18 \\
\hline Tabaquismo & 11 & 17 \\
\hline Inmunosupresión & 10 & 15 \\
\hline EPOC & 7 & 11 \\
\hline Enfermedad cardiaca & 7 & 11 \\
\hline Otra comorbilidad & 7 & 11 \\
\hline Asma & 2 & 3 \\
\hline VIH/sida & 1 & 2 \\
\hline
\end{tabular}

EPOC = enfermedad pulmonar obstructiva crónica, $\mathrm{VIH} / \mathrm{sida}=$ virus de inmunodeficiencia humana/síndrome de inmunodeficiencia adquirida.

día 13 desde el comienzo de síntomas y la defunción se registró el día 17.

En la Tabla 3 se describen las defunciones con antecedente de vacunación, sin comorbilidades y con tratamiento con oseltamivir, aunque de todos se desconoció la fecha de inicio del tratamiento.

\section{Discusión}

Dado que el SISVEFLU capta $100 \%$ de los casos de infección respiratoria aguda grave, las 3089 defunciones notificadas se consideran el universo de las defunciones por influenza, aun cuando un tercio de las infecciones es asintomática y los casos tienen que cumplir criterios sujetos a vigilancia, como la definición operacional. Que únicamente 65 defunciones (2.1\% del total) tuviera antecedente de vacunación con la vacuna sugerida para la temporada y al menos 21 días desde la vacunación hasta el inicio de los síntomas, sugiere que la inmunización contra la influenza estacional es una estrategia rentable, especialmente cuando se considera la inmunidad de grupo, y que se ha comprobado reducción de la carga de la enfermedad y, por lo tanto, de costos de la atención en salud y costos sociodemográficos. ${ }^{12}$

Se ha descrito que la probabilidad de que la vacuna sea costo-efectiva para evitar la muerte es de $100 \%$ y para evitar complicaciones de $96.7 \% .^{12}$

La frecuencia de las defunciones por influenza con antecedente de vacunación fue coincidente con la 
Tabla 3. Descripción de las defunciones por influenza con antecedente de vacunación, sin comorbilidades y con tratamiento antiviral

\begin{tabular}{|c|c|c|c|c|c|c|c|}
\hline Caso & $\begin{array}{l}\text { Edad } \\
\text { sexo }\end{array}$ & Ocupación & Inicio súbito & $\begin{array}{l}\text { Cumple definición } \\
\text { operacional }\end{array}$ & $\begin{array}{l}\text { Vacunación hasta inicio } \\
\text { de síntomas (dias) }\end{array}$ & $\begin{array}{c}\text { Inicio de síntomas } \\
\text { hasta defunción (dias) }\end{array}$ & Subtipo \\
\hline 1 & $\begin{array}{l}1 \\
\text { Femenino }\end{array}$ & - & No & ETI & 57 & 18 & $\begin{array}{l}\text { A H1N1 } \\
\text { PMD }\end{array}$ \\
\hline 2 & $\begin{array}{l}2 \\
\text { Masculino }\end{array}$ & - & Sí & ETI e IRAG & 93 & 3 & A H3 \\
\hline 3 & $\begin{array}{l}38 \\
\text { Femenino }\end{array}$ & Hogar & No & ETI & 50 & - & $\begin{array}{l}\text { A H1N1 } \\
\text { PMD }\end{array}$ \\
\hline 4 & $\begin{array}{l}46 \\
\text { Femenino }\end{array}$ & Hogar & No & IRAG & 66 & 23 & $\begin{array}{l}\text { A H1N1 } \\
\text { PMD }\end{array}$ \\
\hline 5 & $\begin{array}{l}82 \\
\text { Femenino }\end{array}$ & Hogar & Sí & ETI e IRAG & 51 & 10 & A H3 \\
\hline 6 & $\begin{array}{l}86 \\
\text { Femenino }\end{array}$ & Hogar & Sí & ETI e IRAG & 44 & 4 & A H3 \\
\hline
\end{tabular}

ocurrencia de casos en las temporadas; la temporada 2013-2014 fue la que mayor número de defunciones registró. ${ }^{13}$ Lo anterior sugiere que las defunciones por influenza pudieron estar ocasionadas por complicaciones y situaciones que agravaron los casos; individualizar la investigación de cada defunción sería objeto de otro estudio.

La definición operacional de ETI o IRAG se utiliza con fines de vigilancia epidemiológica y ha demostrado ser sensible. En la práctica, el médico debe iniciar el tratamiento antiviral en las primeras 48 horas en los pacientes con tos y fiebre cuando se conoce que los virus de influenza están circulando en la comunidad, ${ }^{14}$ es decir, que un caso no cumpla con la definición operacional no es motivo para retrasar el tratamiento antiviral.

Como se ha descrito en la literatura y reportado por el Sistema Nacional de Vigilancia Epidemiológica, la mayoría de las defunciones por influenza tiene al menos una comorbilidad que agrava la historia natural de la enfermedad. En este estudio, $83 \%$ de los casos presentaba al menos una comorbilidad, como sobrepeso, obesidad, diabetes o insuficiencia cardiaca congestiva, lo que pudo desencadenar una baja respuesta del sistema inmune del hospedero. Desde la pandemia de 2009, la obesidad fue reconocida como un factor de riesgo para las complicaciones de la influenza, esto es importante ya que en México $30 \%$ de los menores, $40 \%$ de los adolescentes y $70 \%$ de los adultos tienen sobrepeso u obesidad, ${ }^{15}$ por lo que nos enfrentamos a nuevos retos en prevención de enfermedades, ya que los adultos obesos con antecedente de vacunación tienen hasta dos veces más riesgo de desarrollar influenza debido a pobre función de las células T.16
La ocurrencia de solo 10 defunciones en pacientes con inmunosupresión en un universo de 3089 defunciones siguiere también un logro en el país respecto al manejo de los pacientes que viven con infección por el virus de la inmunodeficiencia humana y adecuado cumplimiento de las recomendaciones de vacunación en individuos con inmunodepresión. ${ }^{17}$

Las personas que viven con diabetes son más susceptibles a contraer la infección por influenza; también se ha documentado que las enfermedades cardiovasculares asociadas con la influenza pueden llevar a compromiso hemodinámico significativo que requiere soporte cardiaco. ${ }^{18}$

Es de importancia para la salud pública especificar las otras comorbilidades que se presentaron en estas defunciones y que el sistema no permita la aclaración para definir nuevos grupos de riesgo y estrategias de vacunación.

Existe evidencia sólida y consistente de que la vacunación durante el embarazo protege a las mujeres y a sus recién nacidos contra la infección por influenza. ${ }^{19}$ No haber encontrado defunciones en el país de mujeres embarazadas con antecedente de vacunación para la temporada indica la excelente adherencia al objetivo de vacunación en cualquier trimestre del embarazo. ${ }^{17}$

De las seis defunciones por influenza con antecedente de vacunación sin comorbilidades y que recibieron tratamiento antiviral, cuatro correspondieron a individuos en extremos de la vida, lo que concuerda con el conocimiento de que el sistema inmune puede estar inmaduro en los menores o en decadencia en los adultos mayores. ${ }^{20}$ 


\section{Conclusiones}

De acuerdo con los resultados, las defunciones por influenza en pacientes con antecedente de vacunación representan un porcentaje muy bajo del total de las defunciones, únicamente $2.1 \%$.

La existencia de comorbilidades y la pertenencia a los grupos de edad en los extremos de la vida contribuyen a la mortalidad por influenza.

Desde su implementación, la vacunación contra influenza ha sido una estrategia de prevención específica que ha disminuido la carga de la enfermedad en la población general sana y la mortalidad en poblaciones específicas. Este componente del Programa de Vacunación Universal es crucial para proteger a la población mexicana de una de las enfermedades transmisibles más importantes de nuestra era.

La reducción de la mortalidad por influenza es el resultado del alcance de la promoción de la salud, la fortaleza del Sistema de Vigilancia Epidemiológica, la garantía en el abasto de tratamiento y, sobre todo, la consistencia del programa de vacunación.

\section{Agradecimientos}

A la Dirección General de Epidemiología, doctores Cuitláhuac Ruiz Matus, María Eugenia Jiménez Corona y José Alberto Díaz Quiñonez, responsables de la operación de SISVEFLU a nivel federal, estatal y local, por la información y personal de la Red Nacional de Laboratorios Estatales de Salud Pública.

\section{Declaración de conflictos de intereses y financiamiento}

Los autores declaran no tener ningún conflicto de interés. Tampoco existió algún tipo de financiamiento para la realización de este trabajo.

\section{Bibliografía}

1. Fact Sheet: Flu [en línea]. Suiza: Organización Mundial de la Salud; 2018.

2. Hutchinson EC. Influenza virus. Trends Microbiol. 2018;26:809-810,

3. Antecedentes del ACIP 2017-2018 [en línea]. EE. UU.: Centers for Disease Control and Prevention; 2018.

4. Nichol KL. Heterogeneity of influenza case definitions and implications for interpreting and comparing study results. Vaccine. 2006;10:6726-6728.

5. Petrie JG, Ohmit SE, Johnson E, Truscon R, Monto AS. Persistence of antibodies to influenza hemagglutinin and neuraminidase following one or two years of influenza vaccination. J Infect Dis. 2015;212:1914-1922.

6. Skowronski DM, Tweed SA, De Serres G. Rapid decline of influenza vaccine-induced antibody in the elderly: is it real, or is it relevant? J Infect Dis. 2008;197(4):490-502.

7. Castilla J, Martínez-Baz I, Martínez-Artola V, Reina G, Pozo F, García-Cenoz $M$, et al. Decline in influenza vaccine effectiveness with time after vaccination, Navarre, Spain, season 2011/12. Euro Surveill. 2013;18:20388.

8. Pebody R, Andrews N, McMenamin J, Durnall H, Ellis J, Thompson Cl, et al. Vaccine effectiveness of 2011/12 trivalent seasonal influenza vaccine in preventing laboratory-confirmed influenza in primary care in the United Kingdom: evidence of waning intra-seasonal protection. Euro Surveill. 2013;18:20389.

9. Fiore AE, Fry A, Shay D, Gubareva L, Bresee JS, Uyeki TM, et al. Antiviral agents for the treatment and chemoprophylaxis of influenza: recommendations of the Advisory Committee on Immunization Practices (ACIP). MMWR Recomm Rep. 2011;60:1-24.

10. Manual para la vigilancia epidemiológica de influenza. México: Secretaría de Salud/Dirección General de Epidemiología; 2014.

11. Manual de procedimientos estandarizados para el sistema estadístico epidemiológico de las defunciones (SEED). México: Secretaría de Salud/ Dirección General de Epidemiología; 2012.

12. Yang KC, Hung HF, Chen MK, Chen SL, Fann JC, Chiu SY, et al. Cost-effectiveness analysis of universal influenza vaccination: application of the susceptible-infectious-complication-recovery model. Int J Infect Dis. 2018;73:102-108.

13. Ruiz-Matus C, Kuri-Morales $P$, Narro-Robles J. Behavior of influenza seasons in Mexico from 2010 to 2016: analysis and prospective. Gac Med Mex. 2017;153:205-213.

14. Yang JH, Huang PY, Shie SS, Yang S, Tsao KC, Wu TL, et al. Predictive symptoms and signs of laboratory-confirmed influenza: a prospective surveillance study of two metropolitan areas in Taiwan. Medicine (Baltimore). 2015;94:e1952.

15. Encuesta Nacional de Salud y Nutrición de Medio Camino 2016. Informe final de resultados. México: Instituto Nacional de Salud Pública; 2016.

16. Neidich SD, Green WD, Rebeles J, Karlsson EA, Schultz-Cherry S, Noah TL, et al. Increased risk of influenza among vaccinated adults who are obese. Int J Obes (Lond). 2017;41:1324-1330.

17. Lineamientos Generales del Programa de Vacunación Universal y Semanas Nacionales de Salud 2018. [en línea]. México: Secretaría de Salud/Centro Nacional para la Salud de la Infancia y Adolescencia; 2018.

18. Sellers SA, Hagan RS, Hayden FG, Fischer WA. The hidden burden of influenza: A review of the extralpulmonary complications of influenza infection. Influenza Other Respir Viruses. 2017;11:372-393.

19. Mazagatos C, Delgado-Sanz C, Oliva J, Gherasim A, Larrauri A, Spanish Influenza Surveillance System. Exploring the risk of severe outcomes and the role of seasonal influenza vaccination in pregnant women hospitalized with confirmed influenza, Spain, 2010/11-2015/16. PLoS ONE. 2018;13:e0200934.

20. Memoli MJ, Athota R, Reed S, Czajkowski L, Bristol T, Proudfoot K, et al. The natural history of influenza infection in the severely immunocompromised vs nonimmunocompromised hosts. Clin Infect Dis. 2014;58:214-224. 